PROCEEDINGS OF THE

AMERICAN MATHEMATICAL SOCIETY

Volume 140, Number 5, May 2012, Pages 1669-1680

S 0002-9939(2011)11008-6

Article electronically published on September 1,2011

\title{
ON A DISCRETE VERSION OF TANAKA'S THEOREM FOR MAXIMAL FUNCTIONS
}

\author{
JONATHAN BOBER, EMANUEL CARNEIRO, KEVIN HUGHES, AND LILLIAN B. PIERCE
}

(Communicated by Thomas Schlumprecht)

\begin{abstract}
In this paper we prove a discrete version of Tanaka's theorem for the Hardy-Littlewood maximal operator in dimension $n=1$, both in the noncentered and centered cases. For the non-centered maximal operator $\widetilde{M}$ we prove that, given a function $f: \mathbb{Z} \rightarrow \mathbb{R}$ of bounded variation,

$$
\operatorname{Var}(\widetilde{M} f) \leq \operatorname{Var}(f)
$$

where $\operatorname{Var}(f)$ represents the total variation of $f$. For the centered maximal operator $M$ we prove that, given a function $f: \mathbb{Z} \rightarrow \mathbb{R}$ such that $f \in \ell^{1}(\mathbb{Z})$,

$$
\operatorname{Var}(M f) \leq C\|f\|_{\ell^{1}(\mathbb{Z})} .
$$
\end{abstract}

This provides a positive solution to a question of Hajłasz and Onninen in the discrete one-dimensional case.

\section{INTRODUCTION}

It is natural to expect that an averaging operator should have certain smoothing properties; for instance, the spherical means on $\mathbb{R}^{d}$ map $L^{2}$ to $W^{\frac{d-1}{2}, 2}$ 15. Chapter $8, \S 5.21]$. So one could expect that a maximal operator, being a supremum over averages, should not behave too differently. In fact, if maximal operators are not smoothing operators, at least they do not destroy the regularity of functions, up to one weak derivative. This is the principle behind the program started in 1998 by Kinnunen [9] that studies the regularity of maximal operators acting on Sobolev functions. Since then, many authors have contributed to extend the theory, for instance [4, 5], 10], 11, 12, always having in the background the general principle that, for maximal operators, an $L^{p}$-bound implies a $W^{1, p}$-bound.

Things become more difficult when one works with $L^{1}$-functions, since the HardyLittlewood maximal operator does not map $L^{1}$ to $L^{1}$. For $f \in L_{l o c}^{1}\left(\mathbb{R}^{n}\right)$ we define the centered maximal operator as follows:

$$
\mathcal{M} f(x)=\sup _{r>0} \frac{1}{m(B(x, r))} \int_{B(x, r)}|f(y)| \mathrm{d} y,
$$

where $B(x, r)$ is the ball in $\mathbb{R}^{n}$ centered at $x$ with radius $r$ and $m(B(x, r))$ is the $n$-dimensional Lebesgue measure of this ball. In 2004, Hajłasz and Onninen 6, Question 1] asked the following question:

Received by the editors May 6, 2010 and, in revised form, January 14, 2011.

2010 Mathematics Subject Classification. Primary 42B25, 46E35.

Key words and phrases. Maximal operators, Sobolev spaces, discrete operators, Tanaka's theorem.

(C)2011 American Mathematical Society Reverts to public domain 28 years from publication 
Question A. Is the operator $f \mapsto|\nabla \mathcal{M} f|$ bounded from $W^{1,1}\left(\mathbb{R}^{n}\right)$ to $L^{1}\left(\mathbb{R}^{n}\right)$ ?

Observe that by dilation invariance, a bound of the type

$$
\|\nabla \mathcal{M} f\|_{L^{1}\left(\mathbb{R}^{n}\right)} \leq C\left(\|f\|_{L^{1}\left(\mathbb{R}^{n}\right)}+\|\nabla f\|_{L^{1}\left(\mathbb{R}^{n}\right)}\right)
$$

implies that

$$
\|\nabla \mathcal{M} f\|_{L^{1}\left(\mathbb{R}^{n}\right)} \leq C\|\nabla f\|_{L^{1}\left(\mathbb{R}^{n}\right)},
$$

and thus the fundamental question here is to compare the variation of the maximal function with the variation of the original function (perhaps having the additional information that $f \in L^{1}\left(\mathbb{R}^{n}\right)$ ).

In Tanaka's elegant paper [19, he gave a positive answer to this question for the non-centered maximal operator in dimension $n=1$. For $f \in L_{l o c}^{1}\left(\mathbb{R}^{n}\right)$ the non-centered maximal operator is defined as follows:

$$
\widetilde{\mathcal{M}} f(x)=\sup _{\substack{r>0 \\ x \in B_{r}}} \frac{1}{m\left(B_{r}\right)} \int_{B_{r}}|f(y)| \mathrm{d} y,
$$

where the supremum is now taken over all balls $B_{r}$ simply containing $x$. Tanaka established (1.1) for $\widetilde{\mathcal{M}}$ when $f \in W^{1,1}(\mathbb{R})$, with constant $C=2$. This result was later refined by Aldaz and Pérez Lázaro [1, Theorem 2.5], who obtained the sharp constant $C=1$, under only the assumption that $f$ has bounded variation.

Philosophically, the non-centered version is a smoother operator than the centered version since it contains more averages, making it easier to handle. Nevertheless, we expect that (1.1) should also hold in the case of the centered maximal operator with constant $C=1$, when $n=1$ (one may take $f(x)=\chi_{[a, b]}(x)$ for an extremal example). So far, however, Question A remains untouched for the centered version, even in the case $n=1$.

1.1. The discrete one-dimensional setting. Finding discrete analogues for $L^{p}$ bounds in harmonic analysis is a topic of ongoing research. In the simplest cases, $\ell^{p}$-bounds for discrete analogues of classical operators such as Calderón-Zygmund singular integral operators, fractional integral operators, and the Hardy-Littlewood maximal function follow from known $L^{p}$-bounds for the original operators in the Euclidean setting, via elementary comparison arguments (see [16, [17]). But $\ell^{p}$ bounds for discrete analogues of more complicated operators, such as singular, fractional, and maximal Radon transforms (involving integration over a submanifold, or family of submanifolds), are not implied by results in the continuous setting, and moreover the discrete analogues are resistant to conventional methods. Indeed, discrete operators may even behave differently from their continuous counterparts, as is exhibited by the discrete spherical maximal operator [13. It is only recently that substantial progress has been made on discrete operators with Radon characteristics via techniques motivated by the circle method of Hardy and Littlewood, a technique from number theory pioneered in the context of discrete analogues by Bourgain [2, 3] and further developed in a number of interesting cases (see for example [7, [8, 13, 14, 16, 17]).

In this paper we introduce the study of the regularity theory of discrete maximal operators in one dimension. Let $f: \mathbb{Z} \rightarrow \mathbb{R}$ be a discrete function and let $\mathbb{Z}^{+}=\{0,1,2,3, \ldots$,$\} . The discrete centered Hardy-Littlewood maximal operator$ 
is defined by

$$
M f(n)=\sup _{r \in \mathbb{Z}^{+}} \frac{1}{(2 r+1)} \sum_{k=-r}^{k=r}|f(n+k)|,
$$

while the non-centered version is defined by

$$
\widetilde{M} f(n)=\sup _{r, s \in \mathbb{Z}^{+}} \frac{1}{(r+s+1)} \sum_{k=-r}^{k=s}|f(n+k)| .
$$

Our aim is to answer discrete analogues of Question A for these operators. They clearly do not belong to the Radon transform paradigm, and we will not call upon the circle method; instead the challenge arises, at least in the case of the centered maximal operator $M$, from the fact that the analogous result in the continuous setting is not yet known!

In order to study regularity properties of discrete operators, we establish the following conventions. For $1 \leq p<\infty$, the $\ell^{p}$-norm of a function $f: \mathbb{Z} \rightarrow \mathbb{R}$ is

$$
\|f\|_{\ell^{p}(\mathbb{Z})}=\left(\sum_{n=-\infty}^{\infty}|f(n)|^{p}\right)^{1 / p},
$$

and the $\ell^{\infty}$-norm is

$$
\|f\|_{\ell^{\infty}(\mathbb{Z})}=\sup _{n \in \mathbb{Z}}|f(n)| .
$$

We define the derivatives of a discrete function by

$$
\begin{aligned}
f^{\prime}(n) & =f(n+1)-f(n), \\
f^{\prime \prime}(n) & =f(n+2)-2 f(n+1)+f(n), \\
f^{\prime \prime \prime}(n) & =f(n+3)-3 f(n+2)+3 f(n+1)-f(n),
\end{aligned}
$$

and so on. The space corresponding to $W^{k, p}(\mathbb{R})$ is then defined to be the set of discrete functions with finite $w^{k, p}(\mathbb{Z})$-norm where

$$
\|f\|_{w^{k, p}(\mathbb{Z})}=\sum_{j=0}^{k}\left\|f^{(j)}\right\|_{\ell^{p}(\mathbb{Z})} .
$$

But note that by the triangle inequality, for any $k \geq 1$,

$$
\left\|f^{(k)}\right\|_{\ell^{p}(\mathbb{Z})} \leq 2^{k}\|f\|_{\ell^{p}(\mathbb{Z})}
$$

thus in the discrete setting, any $\ell^{p}$-bound automatically provides a $w^{k, p}$-bound, for any $k \geq 1$ (in fact, the discrete $w^{k, p}$-spaces are just the classical $\ell^{p}$-spaces with an equivalent norm). This might make our efforts to transfer the regularity theory for maximal operators to the discrete setting seem almost vacuous.

However, the situation is highly nontrivial when we deal with $\ell^{1}$-functions or functions of bounded variation. We define the total variation of $f: \mathbb{Z} \rightarrow \mathbb{R}$ by

$$
\operatorname{Var}(f)=\left\|f^{\prime}\right\|_{\ell^{1}(\mathbb{Z})}=\sum_{n=-\infty}^{\infty}|f(n+1)-f(n)| .
$$

Our first result, a discrete version of Tanaka's theorem for the discrete non-centered maximal operator with sharp constant, is as follows: 
Theorem 1. Let $f: \mathbb{Z} \rightarrow \mathbb{R}$ be a function of bounded variation. Then

$$
\operatorname{Var}(\widetilde{M} f) \leq \operatorname{Var}(f)
$$

and the constant $C=1$ is the best possible.

We shall prove this result in Section 2, adapting some of the ideas of the original proof of Tanaka for the continuous case. It is not hard to see that the constant $C=1$ is best possible in Theorem [1, for it suffices to consider the function

$$
f(n)=\left\{\begin{array}{lc}
1 & \text { if } n=0 \\
0 & \text { otherwise. }
\end{array}\right.
$$

Dealing with the centered maximal operator is a much more subtle and intricate problem. By an extensive analysis of examples we are led to believe that the same bound should hold for the centered maximal operator:

Question B. Let $f: \mathbb{Z} \rightarrow \mathbb{R}$ be a function of bounded variation. Is it true that

$$
\operatorname{Var}(M f) \leq \operatorname{Var}(f) ?
$$

Motivated by Question B, we prove the following result.

Theorem 2. Let $f: \mathbb{Z} \rightarrow \mathbb{R}$ be a function in $\ell^{1}(\mathbb{Z})$. Then

$$
\operatorname{Var}(M f) \leq\left(2+\frac{146}{315}\right)\|f\|_{\ell^{1}(\mathbb{Z})} .
$$

Theorem 2 represents partial progress toward Question B. In fact, from (1.2), inequality (1.4) would imply (1.5) with constant $C=2$, which would be sharp, with an extremal example given by (1.3). The constant we obtain here is slightly bigger, i.e.,

$$
C=\left(2+\frac{146}{315}\right)=2\left(1+\frac{1}{5}+\frac{1}{7}-\frac{1}{9}\right),
$$

and it appears due to combinatorial arguments in our proof (see Lemma 4 below). It is an interesting question whether one can improve this constant towards the conjectured sharp value.

We expect higher-dimensional analogues of these results, both in the continuous and discrete cases, to hold as well (see the original question by Hajłasz and Onninen [6]). However, the methods of Tanaka [19] and Aldaz and Pérez Lázaro [1 for the one-dimensional continuous (uncentered) case, and ours for the discrete (centered and uncentered) cases do not easily adapt to higher dimensions.

The simplicity and innocence of the objects and statements described above might appear misleading at first glance. Before moving to the proofs, we encourage interested readers to familiarize themselves with the discrete maximal problem, especially Question B above, in order to better appreciate the beauty and the difficulties of the interplay between analysis and combinatorics, still not completely understood, in this problem.

\section{Proof of Theorem 1}

Since $\operatorname{Var}(|f|) \leq \operatorname{Var}(f)$, we may assume without loss of generality that $f$ takes only non-negative values. A function of bounded variation will certainly be bounded and thus, at each point $n$, the averages will also be bounded. However, since we do not assume $f \in \ell^{1}(\mathbb{Z})$, we must be aware of the fact that the supremum over these averages might not be realized. 
We define the left maximal operator as

$$
M_{L} f(n)=\sup _{r \in \mathbb{Z}^{+}} \frac{1}{\left(r+\frac{1}{2}\right)}\left\{\frac{1}{2} f(n)+\sum_{k=-r}^{k=-1} f(n+k)\right\}
$$

and the right maximal operator as

$$
M_{R} f(n)=\sup _{s \in \mathbb{Z}^{+}} \frac{1}{\left(s+\frac{1}{2}\right)}\left\{\frac{1}{2} f(n)+\sum_{k=1}^{k=s} f(n+k)\right\} .
$$

Observe that for each choice of $r, s \in \mathbb{Z}^{+}$we have

$$
\begin{aligned}
\frac{1}{(r+s+1)} \sum_{k=-r}^{k=s} f(n+k)= & \frac{\left(r+\frac{1}{2}\right)}{(r+s+1)}\left(\frac{1}{\left(r+\frac{1}{2}\right)}\left\{\frac{1}{2} f(n)+\sum_{k=-r}^{k=-1} f(n+k)\right\}\right) \\
& +\frac{\left(s+\frac{1}{2}\right)}{(r+s+1)}\left(\frac{1}{\left(s+\frac{1}{2}\right)}\left\{\frac{1}{2} f(n)+\sum_{k=1}^{k=s} f(n+k)\right\}\right) \\
\leq & \frac{\left(r+\frac{1}{2}\right)}{(r+s+1)} M_{L} f(n)+\frac{\left(s+\frac{1}{2}\right)}{(r+s+1)} M_{R} f(n) \\
\leq & \max \left\{M_{L} f(n), M_{R} f(n)\right\} .
\end{aligned}
$$

Therefore we have

$$
\widetilde{M} f(n) \leq \max \left\{M_{L} f(n), M_{R} f(n)\right\},
$$

and since the reverse inequality is obvious, we conclude that

$$
\widetilde{M} f(n)=\max \left\{M_{L} f(n), M_{R} f(n)\right\},
$$

for all $n \in \mathbb{Z}$.

We will say that a point $n$ is a local maximum of $f$ if

$$
f(n-1) \leq f(n) \text { and } f(n)>f(n+1) .
$$

Similarly, a point $n$ is a local minimum of $f$ if

$$
f(n-1) \geq f(n) \text { and } f(n)<f(n+1) .
$$

The following lemma identifies a key property of the local maxima of $M_{L} f$ and $M_{R} f$.

Lemma 3. (i) If $n$ is a local maximum of $M_{L} f$, then $M_{L} f(n)=f(n)$.

(ii) If $n$ is a local maximum of $M_{R} f$, then $M_{R} f(n)=f(n)$.

(iii) If $n$ is a local maximum of $\widetilde{M} f$, then $\widetilde{M} f(n)=f(n)$.

Proof. (i) and (ii). It suffices to prove the result for $M_{R} f$; the argument for $M_{L} f$ is analogous. For this we suppose that $M_{R} f(n)>f(n)$ and consider two cases.

Case 1. $M_{R} f(n)$ is attained for some $s \in \mathbb{Z}^{+}$. 
From the assumption that $M_{R} f(n)>f(n)$ we know that $s \geq 1$. Therefore we have

$$
M_{R} f(n)=\frac{1}{\left(s+\frac{1}{2}\right)}\left\{\frac{1}{2} f(n)+f(n+1)+\sum_{k=2}^{k=s} f(n+k)\right\},
$$

in which the last sum may be vacuous. We can consider an average of length $s-1$ for the point $n+1$ to get

$$
M_{R} f(n+1) \geq \frac{1}{\left(s-\frac{1}{2}\right)}\left\{\frac{1}{2} f(n+1)+\sum_{k=2}^{k=s} f(n+k)\right\} .
$$

Suppose that $M_{R} f(n) \geq M_{R} f(n+1) \geq f(n+1)$. Subtracting (2.2) from (2.1) we have

$$
M_{R} f(n) \leq\left(s+\frac{1}{2}\right) M_{R} f(n)-\left(s-\frac{1}{2}\right) M_{R} f(n+1) \leq \frac{1}{2}(f(n)+f(n+1)),
$$

which is a contradiction. Therefore $M_{R} f(n)<M_{R} f(n+1)$ and $n$ is not a local maximum.

Case 2. $M_{R} f(n)$ is not attained for any $s \in \mathbb{Z}^{+}$.

In this case we can actually prove that for any $m \in \mathbb{Z}$ we have $M_{R} f(m) \geq$ $M_{R} f(n)$. For instance, take $m>n$ and let $C$ be a global upper bound for $f$. Given $\epsilon>0$ there is a large $s>0$ such that

$$
\frac{1}{\left(s+\frac{1}{2}\right)}\left\{\frac{1}{2} f(n)+\sum_{k=1}^{k=s} f(n+k)\right\} \geq M_{R} f(n)-\epsilon .
$$

We then consider an average of length $s$ for the point $m$ to get

$$
\begin{aligned}
M_{R} f(m) & \geq \frac{1}{\left(s+\frac{1}{2}\right)}\left\{\frac{1}{2} f(m)+\sum_{k=1}^{k=s} f(m+k)\right\} \\
& \geq\left(M_{R} f(n)-\epsilon\right)-\frac{2 C(m-n)}{\left(s+\frac{1}{2}\right)} .
\end{aligned}
$$

Letting $\epsilon \rightarrow 0$ and correspondingly letting $s \rightarrow \infty$ along an appropriate sequence, it follows that $M_{R} f(m) \geq M_{R} f(n)$. The case $m<n$ is treated analogously. Thus $n$ is not a local maximum.

(iii) We just have to use the fact that

$$
\widetilde{M} f(n)=\max \left\{M_{L} f(n), M_{R} f(n)\right\},
$$

together with parts (i) and (ii), to conclude that if $n$ is a local maximum of $\widetilde{M} f$, then $\widetilde{M} f(n)=f(n)$.

We now finish the proof of Theorem 1 From now on let us consider the alternating sequence of local maxima $\left\{a_{i}\right\}_{i \in \mathbb{Z}}$ and local minima $\left\{b_{i}\right\}_{i \in \mathbb{Z}}$ of $\widetilde{M} f$, satisfying

$$
\ldots<b_{-2}<a_{-2}<b_{-1}<a_{-1}<b_{0}<a_{0}<b_{1}<a_{1}<b_{2}<a_{2}<\ldots
$$


The sequence (2.3) can be finite or infinite depending on the behavior of the tails of $\widetilde{M} f$. Let us consider the different cases.

Case 1. The sequence (2.3) is infinite.

In this case we have

$$
\begin{aligned}
\operatorname{Var}(\widetilde{M} f) & =\sum_{i=-\infty}^{\infty}\left\{\widetilde{M} f\left(a_{i-1}\right)-\widetilde{M} f\left(b_{i}\right)\right\}+\left\{\widetilde{M} f\left(a_{i}\right)-\widetilde{M} f\left(b_{i}\right)\right\} \\
& =\sum_{i=-\infty}^{\infty}\left\{f\left(a_{i-1}\right)-\widetilde{M} f\left(b_{i}\right)\right\}+\left\{f\left(a_{i}\right)-\widetilde{M} f\left(b_{i}\right)\right\} \\
& \leq \sum_{i=-\infty}^{\infty}\left\{f\left(a_{i-1}\right)-f\left(b_{i}\right)\right\}+\left\{f\left(a_{i}\right)-f\left(b_{i}\right)\right\} \leq \operatorname{Var}(f) .
\end{aligned}
$$

Case 2. The sequence (2.3) is finite on one (or both) side(s).

In this case several different behaviors might occur, but they are essentially treated in the same way, using (2.4) and a minor modification in the tail(s). Suppose for instance that $a_{k}$ is the last local maximum. The function $\widetilde{M} f(n)$ must be monotonically non-increasing for $n \geq a_{k}$, and since it is bounded, the limit

$$
\widetilde{M} f(\infty)=\lim _{n \rightarrow \infty} \widetilde{M} f(n)=c
$$

will exist. In this case we will have

$$
\liminf _{n \rightarrow \infty} f(n) \leq c
$$

Below we write

$$
\operatorname{Var}(f)_{[a, b]}=\sum_{n=a}^{b-1}|f(n+1)-f(n)|
$$

for the variation of $f$ on the interval $[a, b]$, where $a$ and $b$ are integers (or possibly $\pm \infty)$. Therefore we have

$$
\begin{aligned}
\operatorname{Var} & (\widetilde{M} f)=\operatorname{Var}(\widetilde{M} f)_{\left[-\infty, a_{k}\right]}+\operatorname{Var}(\widetilde{M} f)_{\left[a_{k}, \infty\right]} \\
& =\sum_{i=-\infty}^{k}\left\{\left(\widetilde{M} f\left(a_{i-1}\right)-\widetilde{M} f\left(b_{i}\right)\right)+\left(\widetilde{M} f\left(a_{i}\right)-\widetilde{M} f\left(b_{i}\right)\right)\right\}+\left(\widetilde{M} f\left(a_{k}\right)-c\right) \\
& =\sum_{i=-\infty}^{k}\left\{\left(f\left(a_{i-1}\right)-\widetilde{M} f\left(b_{i}\right)\right)+\left(f\left(a_{i}\right)-\widetilde{M} f\left(b_{i}\right)\right)\right\}+\left(f\left(a_{k}\right)-c\right) \\
& \leq \sum_{i=-\infty}^{k}\left\{\left(f\left(a_{i-1}\right)-f\left(b_{i}\right)\right)+\left(f\left(a_{i}\right)-f\left(b_{i}\right)\right)\right\}+\left(f\left(a_{k}\right)-c\right) \\
& \leq \operatorname{Var}(f)_{\left[-\infty, a_{k}\right]}+\operatorname{Var}(f)_{\left[a_{k}, \infty\right]}=\operatorname{Var}(f) .
\end{aligned}
$$

The argument for all the other cases is a minor modification of this one. This concludes the proof of Theorem 1 


\section{Proof of Theorem 2}

One can begin consideration of the discrete centered maximal operator by investigating whether Lemma 3, or any natural modification of it, continues to hold. The following example shows that this need not be the case:

$$
f(n)=\left\{\begin{array}{cc}
10 & \text { if } n= \pm 4 \\
0 & \text { otherwise }
\end{array}\right.
$$

One should not expect the local maxima of $M f$ to touch $f$ or even expect that $M f$ should be convex in each interval in which it disconnects from $f$.

Thus new ideas are required to approach this problem. We start again by assuming that $f$ takes only non-negative values and consider the sequence of local maxima $\left\{a_{i}\right\}_{i \in \mathbb{Z}}$ and local minima $\left\{b_{i}\right\}_{i \in \mathbb{Z}}$ of $M f$ satisfying

$$
\ldots<b_{-2}<a_{-2}<b_{-1}<a_{-1}<b_{0}<a_{0}<b_{1}<a_{1}<b_{2}<a_{2}<\ldots .
$$

We have

$$
\operatorname{Var}(M f)=2 \sum_{i=-\infty}^{\infty}\left(M f\left(a_{i}\right)-M f\left(b_{i}\right)\right)
$$

Remark. If the sequence (3.1) terminates on one or both ends, we modify the sum (3.2) accordingly as follows. Since $f \in \ell^{1}(\mathbb{Z})$, we must have $\lim _{n \rightarrow \pm \infty} M f(n)=$ 0 , and this implies that if the sequence terminates, it would terminate with a last maximum $a_{k}$ and/or a first maximum $a_{l}$ (i.e., it would not terminate with a minimum). If there is a first maximum $a_{l}$, we consider

$$
\operatorname{Var}(M f)=2 M f\left(a_{l}\right)+2 \sum_{i=l+1}^{\infty}\left(M f\left(a_{i}\right)-M f\left(b_{i}\right)\right)
$$

and make minor modifications in the argument below; similar modifications apply if there is a last maximum $a_{k}$.

For each local maximum $a_{i}$ we let $r_{i}$ be the smallest radius such that

$$
M f\left(a_{i}\right)=A_{r_{i}} f\left(a_{i}\right)=\frac{1}{\left(2 r_{i}+1\right)} \sum_{k=-r_{i}}^{k=r_{i}} f\left(a_{i}+k\right),
$$

where we denote by $A_{r}$ the averaging operator of radius $r$ (since $f \in \ell^{1}(\mathbb{Z}$ ), this radius exists). For each point $b_{i}$ we consider the average of radius $s_{i}=r_{i}+\left(a_{i}-b_{i}\right)$, and since we have

$$
M f\left(b_{i}\right) \geq A_{s_{i}} f\left(b_{i}\right),
$$

it follows from (3.2), (3.3) and (3.4) that

$$
\operatorname{Var}(M f) \leq 2 \sum_{i=-\infty}^{\infty}\left(A_{r_{i}} f\left(a_{i}\right)-A_{s_{i}} f\left(b_{i}\right)\right) .
$$

Observe that the interval $\left[b_{i}-s_{i}, b_{i}+s_{i}\right]$ contains the interval $\left[a_{i}-r_{i}, a_{i}+r_{i}\right]$ and they both have the same right endpoint. Now we fix an integer $n$ and we will evaluate the maximum contribution that $f(n)$ can give to the sum on the right hand side of (3.5). 
For each $i \in \mathbb{Z}$, if $n \in\left[a_{i}-r_{i}, a_{i}+r_{i}\right]$, then $n \in\left[b_{i}-s_{i}, b_{i}+s_{i}\right]$ and $f(n)$ contributes to $\left(A_{r_{i}} f\left(a_{i}\right)-A_{s_{i}} f\left(b_{i}\right)\right)$ the amount

$$
\frac{f(n)}{2 r_{i}+1}-\frac{f(n)}{2\left(r_{i}+\left(a_{i}-b_{i}\right)\right)+1} .
$$

If $n \notin\left[a_{i}-r_{i}, a_{i}+r_{i}\right]$, the contribution of $f(n)$ to $\left(A_{r_{i}} f\left(a_{i}\right)-A_{s_{i}} f\left(b_{i}\right)\right)$ is zero or even negative and we disregard it. Now observe that if the contribution (3.6) occurs, we must have $r_{i} \geq\left|n-a_{i}\right|$, and therefore one can show that

$$
\begin{aligned}
f(n)\left(\frac{1}{2 r_{i}+1}-\right. & \left.\frac{1}{2\left(r_{i}+\left(a_{i}-b_{i}\right)\right)+1}\right) \\
\leq f(n) & \left(\frac{1}{2\left|n-a_{i}\right|+1}-\frac{1}{2\left(\left|n-a_{i}\right|+\left(a_{i}-b_{i}\right)\right)+1}\right) \\
& \leq f(n)\left(\frac{1}{2\left|n-a_{i}\right|+1}-\frac{1}{2\left(\left|n-a_{i}\right|+\left(a_{i}-a_{i-1}\right)\right)+1}\right),
\end{aligned}
$$

where in the last step we just used the ordering (3.1). If we sum (3.7) over the index $i$, we obtain an upper bound for the total contribution of $f(n)$ to the right hand side of (3.5), namely

$$
2 f(n) \sum_{i=-\infty}^{\infty}\left(\frac{1}{2\left|n-a_{i}\right|+1}-\frac{1}{2\left(\left|n-a_{i}\right|+\left(a_{i}-a_{i-1}\right)\right)+1}\right) .
$$

Theorem 2 will follow if we prove that for any strictly increasing sequence $\left\{a_{i}\right\}_{i \in \mathbb{Z}}$ of integers, the sum in (3.8) is bounded by a universal constant $C$. This is proved in Lemma 4 below. To conclude the proof of Theorem 2 we will ultimately sum the maximum contributions of all $f(n)$ 's to the total variation (3.5) of $M f$ to prove, as desired, that

$$
\operatorname{Var}(M f) \leq 2 C \sum_{n=-\infty}^{\infty} f(n)=2 C\|f\|_{\ell^{1}(\mathbb{Z})}
$$

Lemma 4. Given $n \in \mathbb{Z}$, for any strictly increasing sequence $\left\{a_{i}\right\}_{i \in \mathbb{Z}}$ of integers,

$$
\sum_{i=-\infty}^{\infty}\left(\frac{1}{2\left|n-a_{i}\right|+1}-\frac{1}{2\left(\left|n-a_{i}\right|+\left(a_{i}-a_{i-1}\right)\right)+1}\right) \leq \frac{4}{3}
$$

If furthermore $a_{i}-a_{i-1} \geq 2$ for all $i \in \mathbb{Z}$, the constant $C=\frac{4}{3}$ may be replaced by $C=1+\frac{1}{5}+\frac{1}{7}-\frac{1}{9}$.

Proof. It is sufficient to prove the result for $n=0$ since we can shift any sequence $a_{i} \mapsto a_{i}+n$. For $n=0$ we aim to prove that

$$
S=\sum_{i=-\infty}^{\infty}\left(\frac{1}{2\left|a_{i}\right|+1}-\frac{1}{2\left(\left|a_{i}\right|+\left(a_{i}-a_{i-1}\right)\right)+1}\right) \leq C .
$$


By shifting the indices we can also assume that $a_{-1} \leq 0<a_{0}$. We divide our sum (3.10) into two parts:

$$
\begin{aligned}
S & =\sum_{i=-\infty}^{-1}\left(\frac{1}{-2 a_{i}+1}-\frac{1}{-2 a_{i-1}+1}\right)+\sum_{i=0}^{\infty}\left(\frac{1}{2 a_{i}+1}-\frac{1}{2\left(a_{i}+\left(a_{i}-a_{i-1}\right)\right)+1}\right) \\
& =S_{1}+S_{2} .
\end{aligned}
$$

The first sum $S_{1}$ is a telescoping sum, and we find that

$$
S_{1} \leq \frac{1}{-2 a_{-1}+1} .
$$

(This continues to hold if the sequence terminates to the left as $i \rightarrow-\infty$.)

The second sum is more involved, and we use the following inequality, for integers $m>n \geq 0$ :

$$
\frac{1}{2 m+1}-\frac{1}{2(m+(m-n))+1} \leq \frac{1}{2(n+1)+1}-\frac{1}{2(m+1)+1} .
$$

Inequality (3.11) can be proved simply by clearing denominators and observing that $m \geq n+1$. We then use (3.11) to bound $S_{2}$ as follows:

$$
\begin{aligned}
S_{2} & =\left(\frac{1}{2 a_{0}+1}-\frac{1}{2\left(a_{0}+\left(a_{0}-a_{-1}\right)\right)+1}\right)+\sum_{i=1}^{\infty}\left(\frac{1}{2 a_{i}+1}-\frac{1}{2\left(a_{i}+\left(a_{i}-a_{i-1}\right)\right)+1}\right) \\
& \leq\left(\frac{1}{2 a_{0}+1}-\frac{1}{2\left(a_{0}+\left(a_{0}-a_{-1}\right)\right)+1}\right)+\sum_{i=1}^{\infty}\left(\frac{1}{2\left(a_{i-1}+1\right)+1}-\frac{1}{2\left(a_{i}+1\right)+1}\right) \\
& \leq\left(\frac{1}{2 a_{0}+1}-\frac{1}{2\left(a_{0}+\left(a_{0}-a_{-1}\right)\right)+1}\right)+\frac{1}{2\left(a_{0}+1\right)+1} .
\end{aligned}
$$

(This also continues to hold if the sequence terminates to the right as $i \rightarrow \infty$.) We have thus arrived at

$$
\begin{aligned}
S & =S_{1}+S_{2} \\
& \leq \frac{1}{-2 a_{-1}+1}+\left(\frac{1}{2 a_{0}+1}-\frac{1}{2\left(a_{0}+\left(a_{0}-a_{-1}\right)\right)+1}\right)+\frac{1}{2\left(a_{0}+1\right)+1} .
\end{aligned}
$$

Recall that here $a_{-1} \leq 0<a_{0}$ are integers. For any $0<a_{0}$ it is easy to see that (3.12) is maximized when $a_{-1}=0$. Then a simple analysis of cases yields that $a_{0}=1$ is the maximal choice, proving that (3.12) is bounded by

$$
C=1+\frac{1}{3}=\frac{4}{3} \text {. }
$$

If we impose the condition $a_{0}-a_{-1} \geq 2$, again we can easily see that the maximum of (3.12) occurs when $a_{-1}=-1$ or 0 . An analysis of a few cases confirms that (3.12) is maximized when $a_{-1}=0$ and $a_{0}=2$, giving the upper bound

$$
C=1+\frac{1}{5}+\frac{1}{7}-\frac{1}{9}=1+\frac{73}{315},
$$

and this finishes the proof of the lemma. 
Observe that in the setting of our maximal operator, we have the condition $a_{i}-a_{i-1} \geq 2$ for all $i \in \mathbb{Z}$ (there must be a local minimum between any two consecutive local maxima), and thus we can use Lemma 4 with constant $C$ given by (3.13) in expression (3.9) to conclude that

$$
\operatorname{Var}(M f) \leq 2\left(1+\frac{73}{315}\right)\|f\|_{\ell^{1}(\mathbb{Z})} .
$$

\section{ACKNOWLEDGMENTS}

The authors would like to thank Jeffrey Vaaler, Jean Bourgain, Diego Moreira and Dimitris Koukoulopoulos for helpful comments during the preparation of this work. The first, second and fourth authors acknowledge support from the Institute for Advanced Study and the National Science Foundation under agreement No. DMS-0635607. The second author also acknowledges support from CAPES/FULBRIGHT grant BEX 1710-04-4. The fourth author is also funded by the Simonyi Fund and National Science Foundation grant DMS-0902658.

\section{REFERENCES}

1. J. M. Aldaz and J. Pérez Lázaro, Functions of bounded variation, the derivative of the one dimensional maximal function, and applications to inequalities, Trans. Amer. Math. Soc. 359 (2007), no. 5, 2443-2461. MR2276629 (2008f:42010)

2. J. Bourgain, On the maximal ergodic theorem for certain subsets of the integers, Israel J. Math. 61 (1988) 39-72. MR937581 (89f:28037a)

3. J. Bourgain, On the pointwise ergodic theorem on $L^{p}$ for arithmetic sets, Israel J. Math. 61 (1988) 73-84. MR937582 (89f:28037b)

4. E. Carneiro and D. Moreira, On the regularity of maximal operators, Proc. Amer. Math. Soc. 136 (2008), no. 12, 4395-4404. MR2431055 (2009m:42030)

5. P. Hajłasz and J. Malý, On approximate differentiability of the maximal function, Proc. Amer. Math. Soc. 138 (2010), no. 1, 165-174. MR2550181(2010i:42040)

6. P. Hajłasz and J. Onninen, On boundedness of maximal functions in Sobolev spaces, Ann. Acad. Sci. Fenn. Math. 29 (2004), no. 1, 167-176. MR2041705(2005a:42010)

7. A. D. Ionescu, A. Magyar, E. M. Stein and S. Wainger, Discrete Radon transforms and applications to ergodic theory, Acta Math. 198 (2007) 231-298. MR2318564(2008i:43007)

8. A. D. Ionescu and S. Wainger, $L^{p}$ boundedness of discrete singular Radon transforms, J. Amer. Math. Soc. 19 (2005), no. 2, 357-383. MR2188130 (2006g:42019)

9. J. Kinnunen, The Hardy-Littlewood maximal function of a Sobolev function, Israel J. Math. 100 (1997), 117-124. MR 1469106 (99a:30029)

10. J. Kinnunen and P. Lindqvist, The derivative of the maximal function, J. Reine Angew. Math. 503 (1998), 161-167. MR.1650343 (99j:42027)

11. J. Kinnunen and E. Saksman, Regularity of the fractional maximal function, Bull. London Math. Soc. 35 (2003), no. 4, 529-535. MR1979008 (2004e:42035)

12. H. Luiro, Continuity of the maximal operator in Sobolev spaces, Proc. Amer. Math. Soc. 135 (2007), no. 1, 243-251. MR2280193 (2007i:42021)

13. A. Magyar, E. M. Stein and S. Wainger, Discrete analogues in harmonic analysis: Spherical averages, Ann. Math. (2) 155 (2002), 189-208. MR.1888798 (2003f:42028)

14. L. B. Pierce, Discrete fractional Radon transforms and quadratic forms, to appear in Duke Math. J.

15. E. M. Stein, Harmonic Analysis: Real Variable Methods, Orthogonality, and Oscillatory Integrals, Princeton University Press, 1993. MR 1232192 (95c:42002)

16. E. M. Stein and S. Wainger, Discrete analogues in harmonic analysis. I: $\ell^{2}$ estimates for singular Radon transforms, Amer. J. Math. 121 (1999), 1291-1336. MR1719802|(2000j:42027) 
17. E. M. Stein and S. Wainger, Discrete analogues in harmonic analysis. II: Fractional integration, J. d'Analyse Math. 80 (2000), 335-355. MR1771530 (2001g:42038)

18. E. M. Stein and S. Wainger, Two discrete fractional integral operators revisited, J. d'Analyse Math. 87 (2002), 451-479. MR.1945293 (2004a:39040)

19. H. Tanaka, A remark on the derivative of the one-dimensional Hardy-Littlewood maximal function, Bull. Austral. Math. Soc. 65 (2002), no. 2, 253-258. MR1898539 (2002m:42017)

School of Mathematics, Institute for Advanced Study, Einstein Drive, Princeton, NEW JERSEy 08540

E-mail address: bober@math.ias.edu

Current address: Department of Mathematics, Box 354350, University of Washington, Seattle, Washington 98195-4350

E-mail address: jwbober@uw.edu

School of Mathematics, Institute for Advanced Study, Einstein Drive, Princeton, NEW JERSEY 08540

E-mail address: ecarneiro@math.ias.edu

Current address: Instituto de Matematica Pura e Aplicada-IMPA, Estrada Dona Castorina 110, Rio de Janeiro, RJ, 22460-320, Brazil

E-mail address: carneiro@impa.br

Department of Mathematics, Princeton University, Fine Hall, Washington Road, Princeton, New Jersey 08544

E-mail address: kjhughes@math.princeton.edu

School of Mathematics, Institute for Advanced Study, Einstein Drive, Princeton, NeW JERSEY 08540

E-mail address: lbpierce@math.ias.edu

Current address: Mathematical Institute, 24-29 St Giles, Oxford OX1 3LB, United Kingdom

E-mail address: lillian.pierce@maths.ox.ac.uk 